\title{
Tamización poblacional para la detección temprana del cáncer de próstata: ¿Qué hemos aprendido en los últimos 10 años?
}

\section{Population Screening for Early Detection of Prostate Cancer: ¿What have we Learned in the Last Decade?}

\author{
David Andrés Castañeda-Millán ${ }^{1,2}$ Cesar Andrés Capera-López ${ }^{1,2}$ Gustavo Ramos-Ulloa ${ }^{3}$ \\ Rodolfo Varela-Ramírez ${ }^{1,2,3}$ Wilfredo Donoso-Donoso ${ }^{1,2,4}$
}

1 Unidad de Urología, Facultad de Medicina, Universidad Nacional de Colombia, Bogotá, Colombia

${ }^{2}$ Grupo de investigación e innovación en Urología, Universidad

Address for correspondence David Andrés Castañeda-Millán, MD, Unidad de Urología, Facultad de Medicina, Universidad Nacional de Nacional de Colombia, Bogotá, Colombia

3 Servicio de Urología Oncológica, Instituto Nacional de Cancerología, Bogotá, Colombia

${ }^{4}$ Unidad de Urología, Hospital Universitario Nacional de Colombia, Bogotá, Colombia

Urol Colomb 2019;28:209-215.

\section{Resumen}

Palabras clave

- neoplasias de la próstata

- cribado

- tamizaje masivo

- antígeno específico prostático
Introducción Desde el inicio de la aplicación del cribado para cáncer de próstata basado en el antígeno prostático específico (PSA) hace aproximadamente dos décadas, la controversia sobre los beneficios y desventajas de su uso rutinario ha sido constante. La literatura médica cuenta con múltiples estudios que en ocasiones han revelado resultados contradictorios sobre los posibles beneficios de la tamización con PSA; la tasa de detección de cáncer de próstata indolente detectado parece ser alta y los estudios no demuestran de forma constante los beneficios en términos de reducción de la mortalidad cáncer específica o general. El propósito del presente artículo, es definir a la luz de la literatura médica reciente, la utilidad a nivel poblacional del cribado para cáncer de próstata basado en el antígeno prostático específico.

Materiales y métodos Se realizó una revisión en los buscadores Pubmed, Embase y Lilacs utilizando los términos MesH "Prostatic neoplasms," "early detection of cancer," "mass screening," "prostate specific antigen," "digital rectal examination," "Outcome assesment (Health care)." Se filtró la búsqueda hacia estudios ejecutados en humanos, y/ o metanálisis y revisiones sistemáticas publicados durante los últimos 10 años. Los abstracts fueron valorados por el grupo de autores e incluidos para análisis según su aporte al objetivo principal del estudio. Algunas referencias adicionales fueron añadidas dada su importancia clínica e histórica.

Resultados Se identificaron 23 referencias con la estrategia de búsqueda, se excluyeron del análisis 9 referencias por no aportar datos relevantes para el presente artículo. Se incluyeron para revisión un total de 14 artículos.
DOI https://doi.org/ $10.1055 / \mathrm{s}-0039-1693136$ ISSN 0120-789X. eISSN 2027-0119.
Copyright $($ 2019, Sociedad Colombiana License terms de Urología. Publicado por Thieme Revinter Publicações Ltda., Rio de Janeiro, Brazil. Todos los derechos reservados.

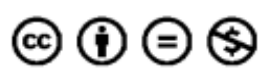




\section{Abstract}

\section{Keywords}

- prostatic neoplasms

- early detection of cancer

- mass screening

- prostate specific antigen
Discusión La tamización para cáncer de próstata con base en el antígeno específico de próstata sérico es una estrategia que permite aumentar la tasa de detección temprana de cáncer, sin embargo, se asocia a una importante tasa de detección de cáncer de próstata indolente y de sobretratamiento. Los resultados de la literatura evaluada son contradictorios con respecto al efecto que tiene la tamización sobre la mortalidad específica por cáncer, algunos estudios han revelado una disminución de ese ítem en los pacientes sometidos a tamización para cáncer de próstata. Los datos también son contundentes en demostrar que las estrategias de tamización no han impactado la supervivencia general en los grupos estudiados. Se esperan resultados de estudios que incluyan el armamento de estrategias disponibles para estimar el riesgo de cáncer de próstata (imágenes y/o nuevos marcadores tumorales) con el fin de mejorar la relación riesgo/beneficio de la estrategia de cribado para cáncer de próstata. Conclusiones La tamización para cáncer de próstata debe ser una estrategia para la detección temprana del cáncer que se usa de forma consensuada con cada paciente y que debe adaptarse al riesgo individual; el paciente a quien se le aplica el cribado debe entender los potenciales riesgos y beneficios de esta estrategia ya que los datos disponibles no permiten demostrar con alto nivel de evidencia, un beneficio clínico traducido en términos de reducción en la mortalidad del cáncer específica o general.

Introduction In the last decade, the prostate-specific antigen based screening for prostate cancer have evoque a lot of controversies on the basis of his risk - benefit ratio; there are controversial data about the impact of this strategy in the male cancer specific and general mortality. The aim of this article is to show the most recent findings and to define the utility of the population screening for early detection of prostate cancer.

Material and Methods A literature review was performed in PubMed, Embase and Lilacs using the MeSH terms: "Prostatic neoplasms," "early detection of cancer," "mass screening," "prostate specific antigen," "digital rectal examination" and "Outcome assesment (Health care)" which was limited to scientific articles published in the past 10 years. The abstracts were evaluated and excluded if they were not related to primary aim of this article. Some references were included given their clinical relevance.

Results 23 articles were retrieved, and after reviewing the abstracts, 9 articles were excluded as they were not related to our primary aim. The analysis was performed in 14 articles.

Discussion The prostate-specific antigen based population screening has been associated with an early diagnosis of prostate cancer even, in a non significative clinical stage, and consequent overtreatment. The literature which was evaluated have controvesial outcomes on the cancer-specific mortality, however none of the articles evaluated shown a significant impact in male general mortality. In the overcoming years, we expect the results of some investigations which includes additional tools for the screening of prostate cancer (multiparametric prostate resonance imaging, new tumoral markers), to improve the risk-benefit of this strategy.

Conclusions The population prostate cancer screening should be made in wellinformed patients and shared based decision process. The urologist and patient should to know the potential benefits and risks of this strategy, because there is not high grade level of evidence which supports a significant effect of this strategy in male general and cancer-specific mortality. 


\section{Introducción}

Actualmente el cáncer de próstata (CaP) es el principal cáncer incidente y la quinta causa de mortalidad por cáncer en hombres; se estima que durante el 2018 se presentaron alrededor de 1,8 millones de casos nuevos de CaP y 359.000 muertes asociadas a CaP alrededor del mundo ${ }^{1}$; en Colombia se estima una incidencia de CaP que oscila entre 27,3-59,7 casos $\times 100.000$ personas/año ${ }^{2}$ y una tasa de mortalidad entre 8,9 y $10,9 \times 100.000$ habitantes hombres. $^{3}$

El uso del antígeno prostático específico (PSA), se introdujo en 1987 para evaluar la respuesta al tratamiento del cáncer de próstata, y posteriormente se adoptó de forma amplia para el cribado poblacional del $\mathrm{CaP}^{4}$ Inicialmente, dos grandes estudios aleatorizados de tamización con PSA: el "Prostate, Lung, Colorectal and Ovarian (PLCO) screening trial" ${ }^{5}$, que incluyó 76685 pacientes y el "The European Randomized Study of Screening for Prostate Cancer (ERSPC)"6, que incluyó 182160 pacientes, demostraron un aumento en la incidencia de CaP en los grupos que recibieron tamización. En el PLCO el riesgo relativo de CaP incidente en el grupo de tamización fue de 1,12; (IC 95\%, 1,07-1,17) sin embargo no hubo beneficio en términos de reducción mortalidad a los 13 años de seguimiento (RR: 1,09, IC 95\%: 0,87-1,36); el estudio ERSPC mostró un aumento en la incidencia de CaP (RR: 1,63, IC 95\%: 1,57-1,69) y menor riesgo de muerte específica por cáncer en el grupo de tamización después de 11 años de seguimiento (RR: 0,79, IC 95\%: 0,68-0,91). La duración del seguimiento en esos estudios fue de 11 y 13 años, respectivamente; esa franja de tiempo dejó dudas respecto a los posibles resultados en mortalidad, ya que ese desenlace, en el contexto del CaP, suele ser más tardío, en especial con aquellos pacientes que iniciaron el cribado a temprana edad. ${ }^{7}$

Actualmente la evidencia acerca del cribado poblacional para CaP con el PSA es controversial, ya que no existen datos sólidos que demuestren una reducción de la mortalidad y por el contrario se ha descrito a nivel mundial una asociación importante entre la tamización con PSA y el aumento del diagnóstico y posible sobretratamiento de esa enfermedad. ${ }^{8}$
El objetivo del presente artículo es definir a la luz de la evidencia más reciente cuáles son los beneficios derivados del uso rutinario del cribado poblacional para CaP con PSA.

\section{Materiales y Métodos}

Se realizó una revisión en los buscadores Pubmed, Embase y Lilacs utilizando los términos MesH "Prostatic neoplasms," "early detection of cancer," "prostate specific antigen," "digital rectal examination," "mass screening," "Outcome assesment (Health care)." Se filtró la búsqueda hacia estudios clínicos, metanálisis y revisiones sistemáticas ejecutadas durante los últimos 10 años. Los abstracts fueron valorados por el grupo de autores e incluidos para análisis según su aporte al objetivo principal del estudio. Algunas referencias adicionales fueron añadidas dada su importancia clínica e histórica. (-Fig. 1)

\section{Resultados}

Tras la estrategia de búsqueda primaria se obtuvo un total de 23 referencias de las cuales se excluyeron 9 por no aportar datos relevantes para el objetivo del presente artículo. Se incluyen en la revisión los resultados de 14 referencias seleccionadas [5, 6, 9-19, 24].

\section{Discusión}

\section{PSA como Estrategia de Tamización Poblacional ¿Tiene o no Beneficios?}

Ilic y cols publicaron en 2013 una revisión sistemática y metaanálisis cochrane. ${ }^{9}$ Incluyeron para el análisis final 5 ensayos clínicos aleatorizados con un total de 341.342 pacientes entre 45 y 80 años con una duración de seguimiento entre 7 y 20 años. Tres de los estudios incluidos se catalogaron con alto riesgo de sesgos y dos de ellos con bajo riesgo (PLCO y el ERSPC); sin embargo, esos últimos señalan resultados contradictorios. El ERSPC reportó una reducción significativa en la mortalidad específica por CaP (RR: 0,84, IC 95\%: 0,73-0,95) mientras que el PLCO no encontró beneficio
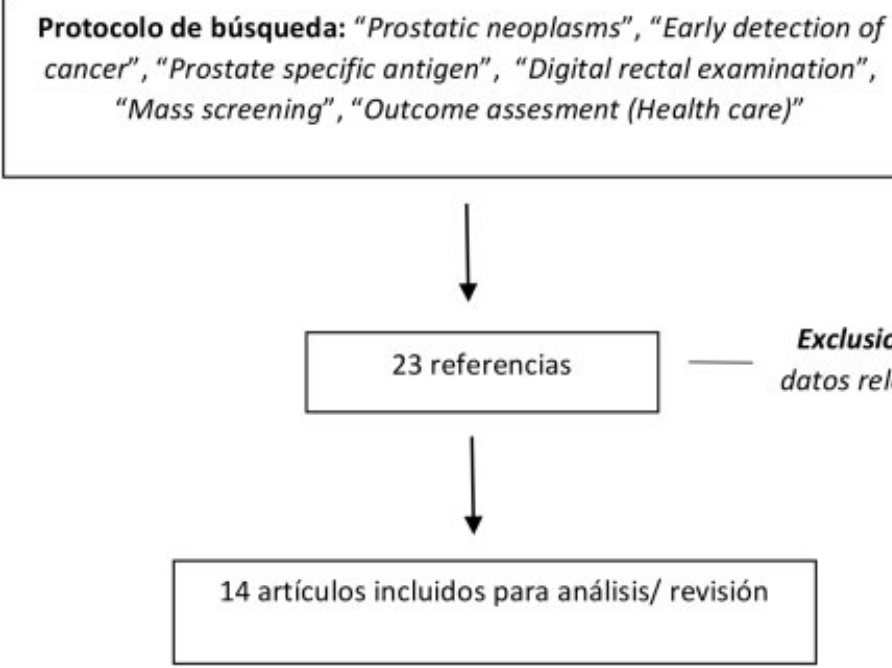

Fig. 1 Protocolo de búsqueda utilizado para la revisión. 
significativo (RR 1,15, IC 95\%: 0,86-1,54). Cuatro de los estudios incluidos en el metaanálisis investigaron la mortalidad por todas las causas y no encontraron una diferencia estadísticamente significativa entre el grupo de tamización vs el grupo control (RR: 1,00, IC 95\%: 0,96-1,03). El diagnóstico de CaP fue mayor en el grupo de tamización (RR 1,30, IC 95\%: CI 1,02-1,65); el CaP localizado fue más comúnmente diagnosticado en el grupo de tamización (RR 1,79, IC 95\%: 1,19-2,70), mientras que el número de pacientes con CaP avanzado fue menor en el grupo de tamización (RR 0,80, IC 95\%: 0,73-0,87). Las conclusiones de esa publicación fueron que: la tamización no disminuye significativamente la mortalidad cáncer específica y se asocia con un alto grado de sobrediagnóstico, tratamiento y complicaciones asociadas al cribado.

Schröder y cols, ${ }^{10}$ ejecutaron un análisis de mortalidad tras 13 años de seguimiento del estudio ERSCP, siendo los resultados más relevantes la reducción significativa en el riesgo de mortalidad específica por CaP en el grupo de tamización RR: 0,83 (IC 95\%: 0,73-0,94, p: 0,004); además mostraron que el NNT (número necesario de tamización) y NND (número necesario diagnóstico) para prevenir una muerte por CaP fueron de 781 y 27 respectivamente y no se encontró diferencia en la mortalidad general en el grupo de tamización (RR: 1,0, IC 95\%: 0,98-1,02, p: 0,98) comparado con el grupo control.

Pinsky y cols ${ }^{11}$ publicaron una actualización del estudio PLCO con una extensión de seguimiento a 14,8 años y a 14,7 años en el grupo de intervención y control respectivamente; se observaron 255 muertes relacionadas con el CaP en el grupo de intervención y 244 muertes en el grupo control (RR mortalidad CaP específica: 1,04, IC 95\%: 0,87-1,24). El RR para mortalidad general fue de 0,97 (IC 95\%: 0,95 -1,004). Esta extensión a 15 años de seguimiento del estudio PLCO, a pesar de la contaminación por la alta tasa de seguimiento con PSA en el grupo control (39,5\% disponía de un PSA en los últimos 3 años y 11,3\% tenía más de una medición de PSA durante los últimos 3 años), continua sin demostrar una reducción significativa en la mortalidad específica por CaP ni en la mortalidad general a pesar de la realización de una estrategia organizada de tamización para CaP.

Carlsson y cols ${ }^{12}$ compararon 3479 pacientes entre 50 y 54 años asignados al grupo de cribado en el estudio de Göteborg iniciado en 1995 con 4060 pacientes entre 50 y 51 años asignados al grupo sin cribado en el estudio preventivo de Malmö ejecutado entre 1982 y 1985; el tiempo promedio de seguimiento fue de 17 años. En el grupo de tamización se encontró una incidencia acumulada de CaP del 15,0\% contra $6,3 \%$ en el grupo control; en el grupo de tamización $76 \%$ de casos incidentes de CaP fueron T1c, mientras que en el grupo control (Malmo) la enfermedad palpable (T2) fue las más frecuente en los casos incidentes (33\%) y se evidenciaron más casos metastásicos al momento del diagnóstico ( $8 \%$ vs $3 \%$ grupo de tamización) [p: $<0,0001]$. El 95\% de los casos incidentes de CaPen la cohorte de Göteborg tenía resultados de biopsia con enfermedad Gleason $\leq 7$, eran más jóvenes (63 vs 67 años) y tenían niveles de PSA menores (media $4,4 \mathrm{ng} / \mathrm{ml}$ vs $12,8 \mathrm{ng} / \mathrm{ml}$ ) que los del grupo de Malmö. El análisis de incidencia demostró que la tamización (grupo de Göteborg) vs no tamización (grupo de Malmö) se asoció a un riesgo mayor de diagnóstico de CaP (IRR: 2,56, IC 95\%: 2,18-3,02), a la disminución en el riesgo de metástasis por CaP (IRR: 0,43, IC 95\%: 0,22-0,79) y de mortalidad cáncer específica (IRR: 0,29, IC 95\%: 0,11-0,67), pero no hubo impacto El NNI (número necesario de inicio de tamización) y el NND (número necesario de diagnóstico) requeridos para prevenir una muerte por CaP luego de 17 años de seguimiento en pacientes que iniciaron cribado entre los 50 y 54 años fueron de 176 y 16 respectivamente. Con base en esos resultados, los autores recomiendan el inicio de la tamización para CaP antes de los 55 años.

Luján y cols ${ }^{13}$ evaluaron la tamización para cáncer de próstata en 2415 hombres españoles de entre 45 y 70 años de edad, con una expectativa de vida mayor a 10 años; con la tamización demostraron un porcentaje mayor de casos incidentes de CaP respecto al grupo control (1861 hombres): $6,7 \%$ [n:162] vs 4,3\% [n: 80] (p: <0,001), con un $88,4 \%$ de casos con enfermedad localizada; sin embargo, en el seguimiento de 15,8 años, no encontraron diferencias en la mortalidad general $(14,1 \%$ vs $14,9 \%)$ ni en la progresión hacia enfermedad avanzada ( $n: 10$ en grupo tamizaje vs $n: 8$ en grupo control. $p=0,938$ ) definida como la aparición de metástasis o PSA $>100 \mathrm{ng} / \mathrm{ml}$. La probabilidad de permanecer libre de diagnóstico de CaP en este estudio fue del $93 \%$ y del $95,8 \%$ para el grupo de tamización vs grupo de control respectivamente.

Martin y cols publicaron en 2018 el "Cluster Randomized Trial of PSA Testing for Prostate Cancer"14; en ese estudio se incluyeron hombres entre 50 y 69 años, de los cuáles 189386 fueron al grupo de tamización con PSA y 219439 al grupo control. El punto de corte de PSA para indicación de biopsia fue de $3 \mathrm{ng} / \mathrm{ml}$ y se alcanzó una adherencia de $40 \%$ y $36 \%$ en el grupo de tamización y control respectivamente. Después de 10 años de seguimiento, se encontró una mortalidad CaP específica en el grupo tamización de 0,30 × 1000 personas /año contra $0,31 \times 1000$ personas/ año en el grupo control (RR: 0,96, IC 95\%: 0,85-1,08, p: 0,50); el porcentaje de pacientes con diagnóstico de CaP fue mayor en el grupo de tamización $(4,3 \%)$ comparado con el grupo control (3,6\%) [RR:1,19, IC 95\%: 1,14-1,25, p: $<0,001]$, el grupo de tamización tuvo una proporción mayor de diagnóstico de CaP Gleason $\leq 6$ vs el grupo control $(3,0 \%$ vs $1,1 \%)$ [6,11 × 1000 hombres, IC 95\%: 5,38-6,84, p: < 0,001] y no se encontraron diferencias en la mortalidad general (RR: 0,99, IC 95\%: 0,94-1,03, p: 0,66).

El "U.S Preventive Service Task Force" publicó una revisión sistemática ${ }^{15}$ sobre la tamización para CaP con base en el PSA; esa publicación encontró que en 2 de los 3 estudios más relevantes (PLCO, ERSCP, CAP) y con mejor calidad disponible en relación al cribado de CaP con PSA, no hubo diferencias en la mortalidad específica por cáncer y que ninguno de esos estudios demostró un impacto significativo en la mortalidad general de los pacientes sometidos a tamización poblacional con PSA (-Tabla 1). Se demostró también que aproximadamente el 32,9\% de biopsias de próstata ejecutadas por elevación de PSA durante el cribado no resultan en diagnóstico de CaP, que el sobrediagnóstico del CaP en los 
Tabla 1 Resultados de tres ensayos clínicos sobre tamización para CaP con PSA

\begin{tabular}{|l|l|l|l|l|}
\hline Estudio & Seguimiento & $\begin{array}{l}\text { Incidencia de CaP } \\
\text { (Tamizaje vs no Tamizaje) }\end{array}$ & $\begin{array}{l}\text { Mortalidad General } \\
\text { (RR: IC 95\%) }\end{array}$ & $\begin{array}{l}\text { Mortalidad Cáncer Específica } \\
\text { (RR: IC 95\%) }\end{array}$ \\
\hline ERSPC [6] & 13 años & $10,2 \% / 6,0 \%$ & $1,00(0,98-1,02)$ & $0,79(0,69-0,91)$ \\
\hline PLCO [5] & 13 y 14,8 años & $11,1 \% / 9,9 \%$ & $0,98(0,95-1,00)$ & $1,04(0,87-1,24)$ \\
\hline CAP [14] & 10 años & $4,3 \% / 3,6 \%$ & $0,99(0,94-1,03)$ & $0,96(0,85-1,08)$ \\
\hline
\end{tabular}

${ }^{*}$ Adaptado de Fenton y cols 15.

brazos de tamización oscila alrededor del $20,7-50,4 \%$ y que los efectos secundarios asociados al diagnóstico (complicaciones postbiopsia transrectal) y tratamiento (disfunción eréctil e incontinencia urinaria), son relevantes en los pacientes que avanzan a fases de estudio o tratamiento por PSA elevado durante el cribado.

Recientemente Osses y cols ${ }^{16}$ publicaron su experiencia con la cohortede Rotterdam del ERSPC. Incluyeron 3331 hombres entre 55 y74 años, los asignaron 1:1 hacia tamización (PSA, TR y ecografía transrectal de próstata cada 4 años) vs grupo control. En el brazo de tamización detectaron 71 casos de CaP contra 57 casos en el grupo control; se detectó enfermedad metastásica en 3 pacientes del grupo de tamización vs 8 en el grupo control. Durante el seguimiento de 19 años el riesgo relativo para progresión a enfermedad metastásica (tamización vs control) fue de 0,42 (IC 95\%: 0,16-1,08), el RR para mortalidad por CaP (tamización vs control) fue 0,47 , IC 95\% (0,14-1,50). La reducción absoluta del riesgo con la tamización para enfermedad metastásica y mortalidad específica por CaP fue de 14,9 (IC 95\%: 2 - 32) y 9,9 (IC 95\%: 5-25) por 1000 hombres, respectivamente. El NNT para evitar una metástasis y una muerte por CaP fue de 67 (IC 95\%: 30ND) y 101 (IC 95\%: 39-ND), respectivamente. Los autores, a pesar de las limitantes estadísticas, postulan que sus resultados demuestran una disminución significativa en el riesgo de progresión a enfermedad metastásica y de mortalidad cáncer específica en los pacientes sometidos a la estrategia de tamización para CaP.

\section{Tacto Rectal: ¿Complemento Primario o Secundario del PSA?}

El cribado para CaP con el tacto rectal (TR) es controvertido debido a la falta de consenso y evidencia de alto nivel que soporte el uso rutinario de esta estrategia.

Naji L y cols ${ }^{17}$ realizaron recientemente una revisión sistemática y metaanálisis sobre el uso del TR como estrategia de tamizaje en el ámbito de la atención primaria que incluyó a 9241 pacientes. Los autores encontraron que el TR para tamizaje del CaP ejecutado por médicos de atención primaria tiene una sensibilidad y especificidad del 51 y $59 \%$ respectivamente. Los estudios analizados se catalogaron con alto riesgo de sesgos, baja calidad y altos niveles de heterogeneidad.

Halpern y cols ${ }^{18}$ analizaron el pronóstico del TR anormal en el grupo de tamizaje del estudio PLCO; entre 35350 pacientes encontraron un TR anormal en 9,2\% (3265) de pacientes y en ese grupo solo el 15,4\% tenían, de forma concurrente, un resultado de PSA anormal ( $>4 \mathrm{ng} / \mathrm{ml}$ ). En el análisis multivariado, encontraron que el TR anormal se asocia con un incremento en la detección de CaP clínicamente significativo (HR-: 2,21, IC 95\%: 1,99-2,44, p: < 0,001) y con la mortalidad cáncer específica (HR: 2,54, IC 95\%: 1,41-4,58, p: 0,002), sin embargo, no se asoció con la mortalidad general (HR: 1,05, IC 95\%: 0.951,15, p: 0,38). En un estudio posterior ${ }^{19}$ se encontró una incidencia acumulativa de CaP clínicamente significativo a los 10 años de seguimiento del 5,9\% (IC 95\%: 5,6-6,2\%) y se demostró la utilidad que puede tener el TR como estrategia de complemento para el tamizaje de CaP en pacientes con niveles de PSA $>3 \mathrm{ng} / \mathrm{ml}$. El incremento en el riesgo absoluto ( $\mathrm{p}:<0,001)$ de presentar CaP a los 10 años de seguimiento con TR anormal, fue del 0,8\% (IC 95\%: 0,4-1,0), 3,0\% (IC 95\%: 1,9-4,0) y del 9,3\% (IC 95\%: 7,9-10) para pacientes con niveles de PSA $<2 \mathrm{ng} / \mathrm{ml}$, entre 2 y $3 \mathrm{ng} / \mathrm{ml}$ y $>3 \mathrm{ng} / \mathrm{ml}$ respectivamente. Los autores proponen que el TR debe ser entendido como una estrategia adyuvante al tamizaje primario con PSA y que debe reservarse para ser aplicado por el urólogo en el contexto de un paciente con PSA elevado.

La organización mundial de la salud ha estipulado que las pruebas de tamización deben tener evidencia científica que demuestre su efectividad y que los beneficios globales de su uso deben superar los riesgos ${ }^{20}$; partiendo de esa premisa, parece ser que el TR como herramienta primaria para la tamización poblacional de CaP, no es una estrategia ideal y debe ser complementaria al PSA en pacientes seleccionados (con PSA elevado).

\section{¿Qué Dicen las Guías Sobre la tamización para CaP?}

La más reciente versión de las guías de la Asociación Europea de Urología ${ }^{21}$ para el cáncer de próstata recomienda con nivel fuerte de evidencia:

- No ejecutar cribado con base en PSA sin ejecutar una asesoría en la que se expliquen los riesgos potenciales y los beneficios.

- Ofrecer una estrategia individualizada y adaptada al riesgo de cada paciente para la detección temprana del $\mathrm{CaP}$ en hombres con buen estatus funcional y con una expectativa de vida no inferior a 10-15 años.

- Ofrecer tamización con PSA a hombres $>50$ años o > 45 años con historia familiar de CaP o de raza afroamericana. 
Las guías de la asociación americana de urología para la detección temprana del CaP revisadas en $2018^{22}$ recomiendan con un nivel moderado de evidencia (nivel B):

- Ejecutar el cribado para CaP con PSA en hombres entre los 55 y 69 años de una forma consensuada, con un proceso que permita la toma de decisiones de forma compartida y en la que se expliquen los riesgos y beneficios de esa estrategia.

Las demás recomendaciones de esta guía tienen un bajo grado de evidencia (nivel C) y dentro de ellas se encuentran:

- No se debe ejecutar tamización para CaP en hombres $<40$ años.

- No se recomienda ejecutar tamización para CaP en hombres de riesgo estándar entre los 40 y 54 años.

- Se recomienda utilizar un periodo de tamización bianual con el propósito de aumentar la proporción de beneficios vs riesgos.

- No se recomienda ejecutar tamización en hombres $>70$ años o con una expectativa de vida inferior a $10-15$ años.

La versión más reciente de las guías para la detección temprana del CaP del "National Comprehensive Cancer Network" (NCCN) ${ }^{23}$ recomiendan con un nivel de evidencia 2B:

- Proponer el cribado para CaP discutiendo los riesgos y beneficios. Ofrecer PSA y TR basal.

- Iniciar la tamización para CaP con PSA en hombres desde los 45 años y hasta los 75 años; considerar un inicio temprano (desde los 40 años), en hombres de raza negra o con mutaciones conocidas del gen BRCA $1 / 2$.

- El TR no debe considerarse como una estrategia de cribado primaria aislada, sino que debe entenderse como una herramienta complementaria en el escenario de un PSA elevado.

- Ejecutar PSA cada 2 a 4 años si el valor inicial es $<1 \mathrm{ng} / \mathrm{ml}$ y cada 1-2 años si el valor inicial oscila entre $1-3 \mathrm{ng} / \mathrm{ml}$ en hombres entre 45 y 75 años.

\section{Hacia el Futuro}

Dadas las limitaciones y riesgos descritos de las estrategias para el cribado del CaP con PSA (sobrediagnóstico, diagnóstico de enfermedad indolente, complicaciones por diagnóstico y tratamiento de cáncer en estadio indolente entre otras), un grupo de investigadores en Finlandia diseño el estudio "ProScreen"24; en este estudio intentarán reclutar 67000 hombres de entre 55 y 67 años aleatorizados 1:3 entre tamización vs grupo control. Los autores pretenden disminuir la tasa de detección de cáncer indolente con el cribado basado en PSA, para tal fin a los pacientes con PSA $>3 \mathrm{ng} / \mathrm{ml}$ les ejecutaran secuencialmente la prueba de 4 calicreínas y si ese resultado es positivo, ejecutarán resonancia multiparamétrica de próstata. La toma de decisiones (biopsia transrectal) se basará en el perfil de riesgo de esos apoyos diagnósticos complementarios. Se espera que los resultados de ese estudio a mediano/largo plazo sean un nuevo insumo para la construcción de una estrategia ideal de tamización poblacional para el CaP.

\section{Conclusiones}

La tamización poblacional para CaP con PSA es una estrategia que aumenta la tasa de detección temprana de CaP, que permite aumentar el diagnóstico de la enfermedad en estados tempranos, que se asocia a un porcentaje elevado de detección de CaP indolente y a cifras importantes de sobretratamiento, que ha demostrado asociarse con una disminución del riesgo para la progresión a enfermedad metastásica y que no ha demostrado tener impacto en la mortalidad general de las poblaciones estudiadas. Los resultados respecto a la mortalidad cáncer específica son contradictorios, y algunos estudios han demostrado una disminución de ese ítem en los grupos con cribado para CaP. El TR no es una estrategia de cribado primario avalada en la atención primaria y debe entenderse como una herramienta coadyuvante al PSA en las estrategias de tamización para CaP. La tamización de CaP debe ser una estrategia para detección temprana del CaP que se usa de forma consensuada con cada paciente y adaptada según el riesgo individual, el paciente a quien se le aplica el tamizaje debe entender los potenciales riesgos y beneficios de esa estrategia.

\section{Financiación}

No se recibió financiación para la elaboración de este manuscrito.

Conflicto de Interés

Ninguno declarado por los autores.

\section{Referencias}

1 Bray F, Ferlay J, Soerjomataram I, Siegel RL, Torre LA, Jemal A. Global cancer statistics 2018: GLOBOCAN estimates of incidence and mortality worldwide for 36 cancers in 185 countries. CA Cancer J Clin 2018;68(06):394-424

2 Bravo LE, Muñoz N. Epidemiology of cancer in Colombia. Colomb Med (Cali) 2018;49(01):9-12

3 Poveda J, Arenas N, Sáenz M, et al. Evolución de la mortalidad por cáncer de próstata en Colombia: estudio ecológico. Urol Colomb 2014;23(01):3-10

4 Barry MJ. Screening for prostate cancer-the controversy that refuses to die. N Engl J Med 2009;360(13):1351-1354

5 Andriole GL, Crawford ED, Grubb RL III, et al; PLCO Project Team. Mortality results from a randomized prostate-cancer screening trial. N Engl J Med 2009;360(13):1310-1319

6 Schröder FH, Hugosson J, Roobol MJ, et al; ERSPC Investigators. Screening and prostate-cancer mortality in a randomized European study. N Engl J Med 2009;360(13):1320-1328

7 Hayes JH, Barry MJ. Screening for prostate cancer with the prostate-specific antigen test: a review of current evidence. JAMA 2014;311(11):1143-1149

8 Stewart RW, Lizama S, Peairs K, Sateia HF, Choi Y. Screening for prostate cancer. Semin Oncol 2017;44(01):47-56

9 Ilic D, Neuberger MM, Djulbegovic M, Dahm P. Screening for prostate cancer. Cochrane Database Syst Rev 2013;31(01):CD004720

10 Schröder FH, Hugosson J, Roobol MJ, et al; ERSPC Investigators. Screening and prostate cancer mortality: results of the European Randomised Study of Screening for Prostate Cancer (ERSPC) at 13 years of follow-up. Lancet 2014;384(9959):2027-2035

11 Pinsky PF, Prorok PC, Yu K, et al. Extended mortality results for prostate cancer screening in the PLCO trial with median follow-up of 15 years. Cancer 2017;123(04):592-599 
12 Carlsson S, Assel M, Ulmert D, et al. Screening for prostate cancer starting at age 50-54 years: A population-based cohort study. Eur Urol 2017;71(01):46-52

13 Luján M, Páez A, AnguloJC, et al. Actualización de los resultados de la rama española del Estudio aleatorizado europeo de screening del cáncer de próstata (ERSPC). Actas Urol Esp 2015;39(07):405-413

14 Martin RM, Donovan JL, Turner EL, et al; CAP Trial Group. Effect of a low-intensity PSA-based screening intervention on prostate cancer mortality: The CAP Randomized Clinical Trial. JAMA 2018; 319(09):883-895

15 Fenton JJ, Weyrich MS, Durbin S, Liu Y, Bang H, Melnikow J. Prostate-specific antigen-based screening for prostate cancer: Evidence report and systematic review for the US preventive services task force. JAMA 2018;319(18):1914-1931

16 Osses DF, Remmers S, Schröder FH, van der Kwast T, Roobol MJ. Results of prostate cancer screening in a unique cohort at $19 \mathrm{yr}$ of follow-up. Eur Urol 2019;75(03):374-377

17 Naji L, Randhawa H, Sohani Z, et al. Digital Rectal Examination for Prostate Cancer Screening in Primary Care: A Systematic Review and Meta-Analysis. Ann Fam Med 2018;16(02):149-154

18 Halpern JA, Shoag JE, Mittal S, et al. Prognostic significance of digital rectal examination and prostate specific antigen in the prostate, lung, colorectal and ovarian (PLCO) cancer screening arm. J Urol 2017;197(02):363-368
19 Halpern JA, Oromendia C, Shoag JE, et al. Use of digital rectal examination as an adjunct to prostate specific antigen in the detection of clinically significant prostate cancer. J Urol 2018;199 (04):947-953

20 Andermann A, Blancquaert I, Beauchamp S, Déry V. Revisiting Wilson and Jungner in the genomic age: a review of screening criteria over the past 40 years. Bull World Health Organ 2008;86 (04):317-319

21 Mottet N, Van den Bergh R, Briers E, et al. EUA, EANM, ESTRO, ESUR, SIOG Guidelines on Prostate Cancer. European association of Urology. Disponible en https://uroweb.org/guideline/prostatecancer/. Acceso en mayo de 2019.

22 Carter H, Albertsen P, Barry M, et al. Early detection of prostate cancer: AUA Guideline. Disponible en https://www.auanet.org/ guidelines/prostate-cancer-early-detection-guideline. Acceso en mayo de 2019.

23 Carrol P, Parson J, Andriole G, et al. NCCN Clinical practice in oncology - Prostate cancer Early detection Version 1.2019. https://www.nccn. org/professionals/physician_gls/pdf/prostate_detection.pdf. Acceso en mayo de 2019.

24 Auvinen A, Rannikko A, Taari K, et al. A randomized trial of early detection of clinically significant prostate cancer (ProScreen): study design and rationale. Eur J Epidemiol 2017;32(06): 521-527 